\title{
QUALIDADE BIOLÓGICA DO SOLO DE Sistemas DE CULTIVO EM POMARES DE Citrus DELICIOSA
}

Henrique Belmonte Petry, Daniele Oliveira, Andressa Oliveira Silveira, Robson Andreazza, Flávio A.O. Camargo

Programa de Pós Graduação em Ciência do Solo - UFRGS; Porto Alegre, RS e-mail: robsonandreazza@yahoo.com.br

\section{Resumo}

A citricultura no Rio Grande do Sul é cultivada sob diversos sistemas de cultivos, sendo que o impacto destes na qualidade do solo é desconhecido. O objetivo deste estudo foi avaliar alguns índices de qualidade ambiental entre os diversos sistemas de manejo de citrus do Vale do Caí, RS, com ênfase na produção de tangerinas 'Montenegrina' (Citrus deliciosa Tenore), através da caracterização química e biológica dos solos. As amostras foram coletadas em cinco áreas cultivadas sob diferentes tipos de cultivo: cultivo orgânico (PO), cultivo orgânico em transição para biodinâmico (POT), cultivo agroflorestal (PAF), cultivo convencional (PC) e mata nativa (MN) como tratamento controle, no município de Montenegro, Rio Grande do $\mathrm{Sul}$, a uma profundidade de $0-10 \mathrm{~cm}$. Foram determinados o carbono da biomassa microbiana (CBM), a respiração basal (RB), as atividades da urease, da fosfatase ácida (FA) e a hidrólise do diacetato de fluoresceína (FDA). Para todas as avaliações, os resultados foram significativamente superiores na área controle $(\mathrm{MN})$. Para o CBM, o tratamento controle (MN) obteve valores de 175,2 $\mathrm{mg} \mathrm{C} \mathrm{Kg}^{-1}$ de solo seco e o menor valor foi encontrado no sistema PC, com $34 \mathrm{mg} \mathrm{C} \mathrm{Kg}^{-1}$ de solo seco. Os valores de RB para o cultivo PAF (334,4 mg C-CO ${ }_{2} \mathrm{~kg}^{-1}$ solo seco), PO (333,3 mg C-CO $\mathrm{kg}^{-1}$ solo seco) e POT (257,7 mg C- $\mathrm{CO}_{2} \mathrm{~kg}^{-1}$ solo seco) foram significativamente menores 
do que os valores de RB do controle (728,7 mg C- $-\mathrm{CO}_{2} \mathrm{~kg}^{-1}$ solo seco). $\mathrm{Na}$ avaliação da urease, os sistemas POT e PO foram significativamente in-

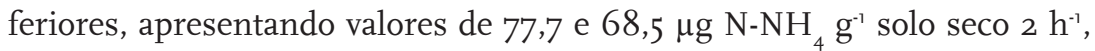
respectivamente, quando comparados ao controle, com valores de $128 \mu \mathrm{g}$ $\mathrm{N}-\mathrm{NH}_{4} \mathrm{~g}_{-1}$ solo seco $2 \mathrm{~h}^{-1}$. Para os sistemas de cultivo PC e PAF, os valores de FA obtidos foram quase três vezes menores (541 e $427 \mu \mathrm{g}$ p-nitrofenol g-1 solo seco $\mathrm{h}^{-1}$, respectivamente) quando comparados com o controle. Portanto, os sistemas de cultivo, mesmo os menos intensivos, não se mostraram capazes de manter a mesma qualidade microbiológica do solo que as áreas controle da região. Isto mostra que indicadores de qualidade do solo como a $\mathrm{RB}$, a FA e a urease podem ser indicadores importantes na avaliação da qualidade ambiental de diferentes sistemas de cultivo de pomares de Citrus deliciosa. Palavras-chave: qualidade ambiental; Citrus deliciosa; sistemas de cultivo; cultivo orgânico.

\section{Abstract}

The citrus production is grown in Rio Grande do Sul under different cropping systems, and their impact on soil quality is unknown. The aim of this study was to evaluate some measures of environmental quality between different management systems of Citrus from Vale do Caí, RS with emphasis on the production of tangerines 'Montenegrina' (Citrus deliciosa Tenore) by chemical and biological characterization of soils. The samples were collected from five areas cultivated under different cropping types: organic cropping (PO), organic cropping in transition to biodynamic (POT), agroforestry cropping $(\mathrm{PAF})$, conventional cropping $(\mathrm{PC})$ and native forestry $(\mathrm{MN})$ as a control treatment in the Montenegro city, Rio Grande do Sul, at o-10 cm of depth. We determined the microbial biomass carbon (CBM), basal respiration (RB), the activities of urease, acid phosphatase (FA) and the hydrolysis of fluorescein diacetate (FDA). For all evaluations, the results were significantly higher in the control area $(\mathrm{MN})$. In CBM evaluations, the control obtained values of $175.2 \mathrm{mg} \mathrm{C} \mathrm{Kg-1} \mathrm{of} \mathrm{dry} \mathrm{soil} \mathrm{and} \mathrm{the} \mathrm{lowest} \mathrm{value} \mathrm{was} \mathrm{found} \mathrm{in} \mathrm{CP} \mathrm{at} 34 \mathrm{mg} \mathrm{C}$ $\mathrm{Kg}^{-1}$ of dry soil. The values of RB to system PAF $\left(334.4 \mathrm{mg} \mathrm{C}^{-\mathrm{CO}_{2}} \mathrm{~kg}^{-1}\right.$ of dry soil), PO (333.3 mg C-CO $\mathrm{kg}^{-1}$ of dry soil) and LPO (257.7 mg C-CO $\mathrm{kg}^{-1}$ soil dried) were significantly lower against the control $\left(728.7 \mathrm{mg} \mathrm{C}^{-\mathrm{CO}_{2}} \mathrm{~kg}^{-1}\right.$ dry soil). In the urease evaluation, the systems POT and PO were significantly lower with values of 77.7 and $68.5 \mu \mathrm{g} \mathrm{N}-\mathrm{NH}_{4} \mathrm{~g}^{-1}$ of dry soil $2 \mathrm{~h}^{-1}$ respectively, compared with the control $\left(128 \mu \mathrm{g} \mathrm{N}-\mathrm{NH}_{4} \mathrm{~g}^{-1}\right.$ of dry soil $\left.2 \mathrm{~h}^{-1}\right)$. For cropping 
systems of PC and PAF, FA values obtained were almost three folds lower (541 and $427 \mu \mathrm{g}$ p-nitrofenol $\mathrm{g}^{-1}$ dry soil $\mathrm{h}^{-1}$ respectively) when compared with the control area. So the cropping systems, even the less intensive, were not able to maintain the same quality of soil microbiology that areas of the native forestry. This shows that soil quality indicators such as RB, the FA and urease can be important indicators in assessing the environmental quality of different cropping systems of Citrus deliciosa.

Keywords: environmental quality; Citrus deliciosa; cropping systems; organic cropping system.

\section{Introdução}

As espécies do gênero Citrus estão entre as espécies frutíferas mais cultivadas no Rio Grande do Sul, predominando o cultivo de laranjas e tangerinas (HAMM, 2005). Segundo Panzenhagen et al. (2008), no Vale do Rio Caí, RS, a citricultura é a principal atividade das unidades de produção, destacando-se a produção de tangerinas 'Montenegrina' (Citrus deliciosa Tenore). Na região, os citros são cultivados sob diversos sistemas de produção, como o orgânico, convencional, produção integrada, agroflorestal, biodinâmico, natural, alternativo e sistemas mistos.

O sistema mais usual é o convencional, o qual não possui regras definidas ou legislação específica (GRUPEX, 2005), tendo como características o uso intensivo de insumos químicos e tráfego intenso de máquinas nos pomares, deixando comumente o solo exposto, com baixa produção de biomassa nas linhas e entrelinhas dos pomares. Os pomares sob sistema orgânico estão regidos pela Lei ${ }^{\circ} 10.831$ (BRASIL, 2003), na qual são considerados todos aqueles que adotam técnicas específicas que tenham como objetivo a sustentabilidade ecológica e econômica, entre outras características.

Segundo Gliessman (2001), o manejo sustentável de agroecossistemas requer o conhecimento de como fatores individuais influenciam nos organismos cultivados e como os fatores interagem para formar o complexo ambiental. Os manejos conservacionistas do solo são aqueles que têm por objetivo manter, controlar ou restaurar as propriedades do recurso alteradas pela degradação (RIENZI e MAGGI, 2007). A matéria orgânica do solo (MOS) é a principal fonte de energia para os microorganismos, de nutrientes para as plantas (principalmente o N) e condiciona o solo dando estabilidade aos agregados, possuindo, assim, função vital na manutenção da qualidade do solo (MOREIRA e SIQUEIRA, 2006).

A transformação e a degradação da MOS ocorrem em decorrência da liberação de enzimas pelos microrganismos que oxidam os compostos orgânicos 
liberando $\mathrm{CO}_{2}$. Dessa forma, a atividade enzimática tem potencial de indicar as transformações biológicas do solo em resposta às mudanças no manejo, mostrando uma relação estreita entre manejo e a microbiota do solo, influenciando na qualidade deste (BUZINARO et al., 2009). A qualidade do solo pode ser mensurada através do uso de indicadores, que são atributos que medem ou refletem o status ambiental ou a condição de sustentabilidade do ecossistema e podem ser classificados como físicos, químicos e biológicos (ARAÚJO e MONTEIRO, 2007). Essa qualidade é definida como a capacidade deste de funcionar dentro do ecossistema para sustentar a produtividade biológica, manter a qualidade ambiental e promover o crescimento das plantas e animais (MOREIRA e SIQUEIRA, 2006).

As alterações em alguns indicadores biológicos do solo pela adoção de diferentes sistemas de manejo foram verificadas por D'Andrea et al. (2002), na região do cerrado do estado de Goiás, e por Santos et al. (2004), no Rio Grande do Sul. Além disso, trabalhos que avaliam o efeito do manejo orgânico na cultura do citrus em comparação com o manejo convencional estão sendo estudados (SORRENTI et al., 2008; BUZINARO et al., 2009; CANALI et al., 2009), mas ainda é necessário intensificar os trabalhos na qualidade ambiental. Em virtude disso, este trabalho teve como objetivo avaliar a qualidade biológica de solos sob diferentes sistemas de manejo em pomares de tangerineiras 'Montenegrina', utilizando como indicadores a biomassa microbiana (CBM), a respiração basal ( $\mathrm{RB} \mathrm{C}-\mathrm{CO}_{2}$ ) e a atividade das enzimas urease, fosfatase ácida (FA) e da hidrólise do diacetato de fluoresceína (FDA).

\section{Materiais e métodos}

\section{Caracterização dos solos}

As áreas em estudo estão localizadas em quatro propriedades de citricultores do município de Montenegro, Estado do Rio Grande do Sul. Os pomares escolhidos para a pesquisa possuem características de relevo e solo semelhantes. Os solos utilizados foram classificados como ARGISSOLO Vermelho Distrófico espessarênico (STRECK et al., 2008), com clima tipo CFa da classificação de Köppen. A amostragem do solo foi realizada em outubro de 2009 , na camada de $0-10 \mathrm{~cm}$, e foram coletadas oito subamostras para formação de uma amostra composta em cada área avaliada. As amostras foram colocadas em sacos 
plásticos e transportadas até o laboratório, onde foram homogeneizadas, divididas em três porções, peneiradas $(2 \mathrm{~mm})$ e refrigeradas a $4^{\circ} \mathrm{C}$, com umidade de campo, até o momento da realização das análises. Foi retirado $20 \mathrm{~g}$ de solo de cada amostra para a determinação do teor de umidade, por secagem em estufa a $105^{\circ} \mathrm{C}$ até peso constante. Uma fração de cada amostra, com aproximadamente $500 \mathrm{~g}$, foi separada para a caracterização química (Tabela 1), conforme metodologias descritas por Tedesco et al., 1995. Foram determinados o carbono da biomassa microbiana (CBM), a respiração basal (RB), atividade das enzimas urease e fosfatase ácida (FA) e a hidrólise do diacetato de fluoresceína (FDA).

Tabela 1. Caracterização físico-química dos solos sob diferentes tipos de cultivo de tangerina: cultivo orgânico (PO), cultivo orgânico em transição para biodinâmico (POT), cultivo agroflorestal (PAF), cultivo convencional (PC) e mata nativa (MN).

\begin{tabular}{|c|c|c|c|c|c|}
\hline & PO & РOT & PC & PAF & MN \\
\hline Teor de argila (\%) & 10 & 11 & 13 & 15 & 13 \\
\hline $\mathrm{pH}$ em $\mathrm{H}_{2} \mathrm{O}$ & 7,5 & 7,4 & 6,1 & 6,3 & 5,9 \\
\hline Índice SMP & 7,5 & 7,4 & 6,1 & 6,3 & 5,9 \\
\hline $\mathrm{P}\left(\mathrm{mg} \cdot \mathrm{kg}^{-1}\right)$ & $>100$ & $>100$ & 92 & 51 & 17 \\
\hline K (mg.kg-1) & 42 & 123 & 115 & 26 & 127 \\
\hline MOS (\%) & 4,2 & 4,2 & 2 & 3,7 & 3,8 \\
\hline Ca troc. $\left(\mathrm{cmol}_{\mathrm{c}} \cdot \mathrm{dm}^{-3}\right)$ & 14,3 & 11,2 & 3,9 & 8,4 & 5 \\
\hline $\mathrm{Mg}$ troc. $\left(\mathrm{cmol}_{\mathrm{c}} \cdot \mathrm{dm}^{-3}\right)$ & 0,7 & 1,1 & 0,8 & 0,9 & 1,2 \\
\hline $\mathrm{Al}+\mathrm{H}\left(\mathrm{cmol}_{c} \cdot \mathrm{dm}^{-3}\right)$ & 0,8 & 0,6 & 2,5 & 2,5 & 3,5 \\
\hline CTC $\left(\mathrm{cmol}_{\mathrm{c}} \cdot \mathrm{dm}^{-3}\right)$ & 15,9 & 13,2 & 7,5 & 11,8 & 10,2 \\
\hline Valor V & 95 & 95 & 67 & 79 & 64 \\
\hline $\mathrm{Ca} / \mathrm{Mg}$ & 20 & 10 & 4,9 & 9 & 4,2 \\
\hline $\mathrm{Ca} / \mathrm{K}$ & 133 & 36 & 13 & 126 & 15 \\
\hline $\mathrm{Mg} / \mathrm{K}$ & 6 & 3,5 & 2,7 & 14 & 3,7 \\
\hline $\mathrm{S}\left(\mathrm{mg} \cdot \mathrm{kg}^{-3}\right)$ & 12 & 12 & 7,5 & 13 & 14 \\
\hline Zn (mg.kg-3) & 27 & 27 & 15 & 12 & 7,6 \\
\hline $\mathrm{Cu}\left(\mathrm{mg} \cdot \mathrm{kg}^{-3}\right)$ & 7,9 & 5,5 & 9,5 & 3,8 & 0,7 \\
\hline B (mg.kg-3) & 0,8 & 0,5 & 0,3 & 0,4 & 0,6 \\
\hline $\mathrm{Mn}\left(\mathrm{mg} \cdot \mathrm{kg}^{-3}\right)$ & 2 & 2 & 25 & 4 & 1 \\
\hline
\end{tabular}


Caracterização das áreas

Foram avaliadas quatro áreas de pomares sob diferentes tipos de cultivo, instalados por citricultores da região de Montenegro e uma área de mata nativa (controle), as quais diferem seus manejos ao longo do ano agrícola, conforme descrição a seguir.

Área 1 (PO) - Sistema de cultivo orgânico, produtor associado à Cooperativa dos Citricultores Ecológicos do Vale do Rio Caí (ECOCITRUS), estando sob manejo orgânico há aproximadamente 13 anos com adubações anuais de composto orgânico sólido e líquido, ambos produzidos pela cooperativa, cuja composição está descrita na Tabela 2. A área está localizada nas coordenadas $29^{\circ} 36^{\prime} 47^{\prime \prime}$ S e $51^{\circ} 29^{\prime} 57^{\prime \prime} O$. O manejo das plantas espontâneas é realizado com roçadas nas entrelinhas e sob a copa das plantas. O controle fitossanitário é feito com aplicações de calda bordalesa e calda sulfocálcica.

Área 2 (POT) - Sistema de cultivo orgânico em transição para biodinâmico, produtor associado à cooperativa ECOCITRUS, estando sob manejo orgânico há aproximadamente seis anos e anteriormente cultivado convencionalmente. A área está localizada nas coordenadas $29^{\circ} 38^{\prime} 11^{\prime \prime S}$ e $51^{\circ} 28^{\prime} 7^{\prime \prime} \mathrm{O}$. Atualmente, não aplica mais os fertilizantes orgânicos da cooperativa e utiliza os compostos da agricultura biodinâmica. O manejo das plantas espontâneas e o controle fitossanitário são realizados conforme o produtor da Área 1.

Área 3 (PAF) - Sistema de cultivo agroflorestal, produtor associado à cooperativa ECOCITRUS, estando sob este manejo há aproximadamente 12 anos, desde que o pomar foi instalado, onde foram consorciadas espécies nativas florestais, como o angico-vermelho (Parapiptadenia rígida Benth). A área situa-se nas coordenadas $29^{\circ} 38^{\prime} 45^{\prime \prime} \mathrm{S}$ e $5^{\circ} 34^{\prime} 18^{\prime \prime} \mathrm{O}$. Na implantação do pomar, foram fertilizadas as áreas com os compostos orgânico e líquido da cooperativa devido à alta degradação da área e, posteriormente, não foram utilizados fertilizantes, utilizando-se somente os resíduos das podas anuais realizadas nas espécies florestais. O manejo das plantas espontâneas é feito com roçadas esporádicas, já que o seu crescimento é reduzido, devido ao alto sombreamento que conferem as espécies florestais. O manejo fitossanitário é realizado com uma pulverização anual de calda bordalesa na floração.

Área 4 (PC) - Sistema de cultivo convencional instalado há aproximadamente 15 anos, com uso intensivo de fertilizantes minerais, herbicidas em área total duas vezes ao ano, na primavera e no verão, e produtos fitossanitários como fungicidas, inseticidas e acaricidas. A área situa-se nas coordenadas $29^{\circ} 37^{\prime} 41^{\prime \prime} \mathrm{S}$ e $51^{\circ} 28^{\prime} 31^{\prime \prime} \mathrm{O}$. 
Área $5(\mathrm{MN})$ - Mata nativa secundária com árvores altas, basicamente Mirtáceas e Fabáceas, com sub-bosque bem desenvolvido, porém com sinais de acesso ocasional de bovinos. Área regenerada há cerca de 30 anos. A área de mata nativa está localizada nas coordenadas $29^{\circ} 39^{\prime} 45^{\prime \prime} \mathrm{S}$ e $51^{\circ} 31^{\prime} 10^{\prime \prime} \mathrm{O}$.

Tabela 2. Caracterização química e física dos compostos sólido e líquido da Cooperativa Ecocitrus.

\begin{tabular}{|c|c|c|}
\hline & Composto sólido & Composto líquido \\
\hline Umidade (\%) & 48 & 91 \\
\hline $\mathrm{pH}$ & 8,7 & 5,9 \\
\hline Carbono Orgânico (\%) & 14 & 42 \\
\hline Nitrogênio (\%) & 1,5 & 2,8 \\
\hline $\mathrm{P}(\%)$ & 0,2 & 1,1 \\
\hline $\mathrm{K}(\%)$ & 0,5 & 0,4 \\
\hline Сa (\%) & 8,0 & 1,7 \\
\hline $\mathrm{Mg}(\%)$ & 0,3 & 0,2 \\
\hline $\mathrm{S}(\%)$ & 0,3 & 0,4 \\
\hline $\mathrm{Cu}\left(\mathrm{mg} \cdot \mathrm{kg}^{-1}\right)$ & 18 & 76 \\
\hline $\mathrm{Zn}\left(\mathrm{mg} \cdot \mathrm{kg}^{-1}\right)$ & 43 & 417 \\
\hline $\mathrm{Fe}(\%)$ & 0,9 & 1,6 \\
\hline $\mathrm{Mn}\left(\mathrm{mg} \cdot \mathrm{kg}^{-1}\right)$ & 346 & 185 \\
\hline $\mathrm{Na}\left(\mathrm{mg} \cdot \mathrm{kg}^{-1}\right)$ & 0,2 & 0,8 \\
\hline $\mathrm{Cd}\left(\mathrm{mg} \cdot \mathrm{kg}^{-1}\right)$ & 0,4 & 0,6 \\
\hline $\mathrm{Cr}\left(\mathrm{mg} \cdot \mathrm{kg}^{-1}\right)$ & 28 & 74 \\
\hline $\mathrm{Ni}\left(\mathrm{mg} \cdot \mathrm{kg}^{-1}\right)$ & 19 & 11 \\
\hline $\mathrm{Pb}\left(\mathrm{mg} \cdot \mathrm{kg}^{-1}\right)$ & 7 & 22 \\
\hline Mo (mg.kg-1) & $<0,2$ & 0,4 \\
\hline $\mathrm{V}\left(\mathrm{mg} \cdot \mathrm{kg}^{-1}\right)$ & 17 & 7 \\
\hline As $\left(\mathrm{mg} \cdot \mathrm{kg}^{-1}\right)$ & $<2$ & 4 \\
\hline $\mathrm{B}\left(\mathrm{mg} \cdot \mathrm{kg}^{-1}\right)$ & 14 & 25 \\
\hline $\mathrm{Hg}\left(\mathrm{mg} \cdot \mathrm{kg}^{-1}\right)$ & 0,01 & $<0,01$ \\
\hline Poder de Neutralização(\%) & 22 & $<1$ \\
\hline
\end{tabular}


Determinação do carbono da biomassa microbiana (CBM) Para a determinação do CBM, foi utilizada a metodologia de Tate et al. (1988). Para esta análise, foram pesadas $20 \mathrm{~g}$ de cada amostra de solo úmido, três amostras foram adicionadas em vidros de extrato para serem fumigadas e três em erlenmeyers (controle, não fumigado). As amostras controle foram extraídas com $50 \mathrm{~mL}^{2} \mathrm{~K}_{2} \mathrm{SO}_{4}(\mathrm{O}, 5 \mathrm{M})$, como descrito abaixo. As outras três amostras foram fumigadas em dessecador (forrado com papel toalha úmido) contendo aproximadamente $25 \mathrm{~mL}$ de clorofórmio purificado (livre de álcool) em um becker pequeno, com pérolas de vidro. O dessecador foi fechado hermeticamente e realizado um vácuo até o borbulhamento do clorofórmio. Posteriormente, foram incubados a $27^{\circ} \mathrm{C}$ por $24 \mathrm{~h}$. Após este período, retirou-se o papel e o becker com clorofórmio e efetuaram-se vácuos sucessivos para retirar o excesso do fumigante. Para a extração, as amostras de solo foram transferidas para erlenmeyers de $125 \mathrm{~mL}$, e foram adicionados $50 \mathrm{~mL}$ de $\mathrm{K}_{2} \mathrm{SO}_{4}$. Agitou-se por 30 minutos e filtrou-se a suspensão resultante (papel de filtro Whatman $n^{\circ} 42$ ). Para a oxidação do carbono, as amostras foram colocadas em tubos de ensaio com o,5 mL de extrato da amostra, 0,5 $\mathrm{mL}$ de água, $1 \mathrm{~mL}$ da solução $\mathrm{Mn}$ (III)-pirofosfato e $1 \mathrm{~mL}$ de ácido sulfúrico concentrado. $\mathrm{O}$ conjunto foi agitado suavemente para a homogeneização e deixado em repouso por 18 horas. Após esse período, foi realizada a leitura em espectrofotômetro $(\lambda=495 \mathrm{~nm})$. A quantidade de carbono nas amostras foi determinada com base na curva padrão preparada com concentrações conhecidas de carbono $(6,12,18,24$ mg de C L-1) (GAMA-RODRIGUES et al., 1997).

\section{Determinação da respiração basal (RB)}

A RB microbiana foi estimada pela quantidade de $\mathrm{CO}_{2}$ liberado do solo durante 20 dias de incubação utilizando-se a metodologia de Stotzky (1972). Foram colocadas $50 \mathrm{~g}$ de solo em um recipiente de vidro de $800 \mathrm{~mL}$ hermeticamente fechado, e este foi incubado a $28^{\circ} \mathrm{C}$, com a umidade ajustada para $75 \%$ de sua capacidade de campo. Foram utilizados três recipientes sem solo, como controle. $\mathrm{O} \mathrm{CO}_{2}$ foi capturado por $20 \mathrm{~mL}$ de uma solução de $\mathrm{NaOH}$ 0,5 M e quantificado por titulação com $\mathrm{HCl}$ o,3 M, sendo adicionados anteriormente $3 \mathrm{~mL}$ de $\mathrm{BaCl} 30 \%$ e utilizada fenolftaleína a $1 \%$ como indicador. Durante esse período, foram realizadas duas titulações, a primeira aos 10 dias e a segunda aos 20 dias após o início da incubação das amostras, sendo os valores somados para se obter o valor referente a 20 dias de incubação. 


\section{Determinação da atividade da Fosfatase Ácida (FA)}

A atividade da FA foi determinada segundo Dick et al. (1996), através da determinação colorimétrica do p-nitrofenol. O substrato utilizado foi o p-nitrofenil fosfato dissódico (PNP 0,05 M). Em cada amostra, foram adicionados 0,25 mL de tolueno, 4 mL de tampão MUB pH 6,5 e o,1 mL de solução PNP $0,5 \mathrm{M}$ por g de solo, incubando-se as amostras a $37^{\circ} \mathrm{C}$ durante uma hora. Após esse período, foram adicionados à amostra 4,0 mL de $\mathrm{NaOH} 0,5 \mathrm{M} \mathrm{e}$ 1, $\mathrm{mL}$ de $\mathrm{CaCl}_{2}$ O,5 M. Após a adição do $\mathrm{CaCl}_{2}$, as amostras foram filtradas em papel filtro Whatman $n^{\circ} 2$. A intensidade da coloração amarela do filtrado foi determinada em espectrofotômetro $(\lambda=410 \mathrm{~nm})$. A quantidade de p-nitrofenol formada em cada amostra foi determinada com base numa curva padrão preparada com concentrações conhecidas de p-nitrofenol (10, 20, 30, 40, $50 \mathrm{mg}$ de p-nitrofenol $\mathrm{mL}^{-1}$ ). A atividade enzimática foi expressa em $\mathrm{mg}$ de p-nitrofenol liberado por hora por grama de solo seco (mg p-nitrofenol $\mathrm{h}^{-1} \mathrm{~g}^{-1}$ solo seco).

\section{Determinação da atividade da urease}

A atividade da urease foi determinada segundo metodologia proposta por Dick et al. (1996) e o substrato utilizado foi uma solução de ureia 0,2 M. Foram adicionados $0,25 \mathrm{~mL}$ de tolueno, 9, $0 \mathrm{~mL}$ da solução THAM pH 9, ० e 1,0 mL da solução de ureia o,2 M em 5,o g de solo. Posteriormente, incubou-se a $37^{\circ} \mathrm{C}$ por duas horas. Após a incubação, foram adicionados $35 \mathrm{~mL}$ de solução gelada de $\mathrm{KCl}-\mathrm{Ag}_{2} \mathrm{SO}_{4}$ misturando-a com a amostra. O conteúdo foi então ajustado para $50 \mathrm{~mL}$ com adição de $\mathrm{KCl}-\mathrm{Ag}_{2} \mathrm{SO}_{4}$. Para determinar $\mathrm{N}-\mathrm{NH}_{4}^{+}$na suspensão de solo resultante, foi retirada uma alíquota de $20 \mathrm{~mL}$ da suspensão, procedeu-se à destilação a vapor desta alíquota com 0,2 g de $\mathrm{MgO}$ por quatro minutos, como descrito por Tedesco et al. (1995) para análi-

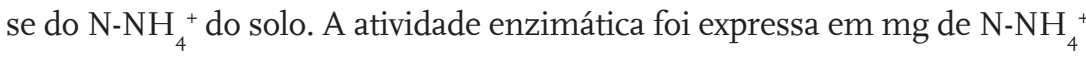
liberado durante duas horas por g de solo ( $\mathrm{mg} \mathrm{N}-\mathrm{NH}_{4}{ }^{+} 2 \mathrm{~h}^{-1} \mathrm{~g}^{-1}$ solo seco).

\section{Determinação da hidrólise do Diacetato de Fluoresceína (FDA)}

A hidrólise do diacetato de fluoresceína foi determinada utilizando-se a metodologia de Green et al. (2006), com algumas alterações descritas a seguir. Foi incubado, em triplicata, $1 \mathrm{~g}$ de solo com $20 \mathrm{~mL}$ de tampão fosfato de sódio $60 \mathrm{mM}$ a $25^{\circ} \mathrm{C}$ durante 15 minutos sob agitação a $100 \mathrm{rpm}$. Após este período, foram adicionados $100 \mu \mathrm{L}$ da solução de FDA 4,8 mM. As 
amostras foram então agitadas por uma hora e 45 minutos (100 rpm, $25^{\circ} \mathrm{C}$ ) e, após, foram adicionados $20 \mathrm{~mL}$ de acetona em cada frasco, e solução de FDA 4,8 mM nas amostras controle (que não receberam esta solução antes da incubação). Foram então centrifugadas a 6.000 rpm por cinco minutos e filtradas em papel filtro Whatman $n^{\circ} 4$. Foi medida a intensidade da cor amarela em espectrofotômetro $(\lambda=490 \mathrm{~nm})$. A concentração de fluoresceína foi calculada com o auxílio de uma curva padrão preparada com concentrações conhecidas de fluoresceína $\left(1,2,3,4,5 \mu \mathrm{g}\right.$ de fluoresceína $\left.\mathrm{mL}^{-1}\right)$. A atividade enzimática foi expressa em $\mu$ g de fluoresceína liberada por grama de solo seco ( $\mu \mathrm{g} \mathrm{F} \mathrm{g}^{-1}$ solo seco) por hora.

\section{Análise estatística}

Para a análise estatística, foi utilizado o programa Statistica 7 (STATSOFT, 2007). Os resultados de atividade enzimática foram submetidos à análise de variância (ANOVA) e as médias de cada atributo dentro de cada local foram comparadas entre si através do teste T de Student, ao nível de significância de 5\% (STATSOFT, 2007).

\section{Resultados e discussão}

Os valores de carbono na biomassa microbiana (CBM) e da respiração basal (RB) foram significativamente maiores no tratamento de mata nativa (MN) (Figura 1). O maior valor de CBM encontrado no sistema MN com 175,2 mg $\mathrm{C} \mathrm{Kg}^{-1}$ de solo seco e o menor valor foi encontrado no sistema de cultivo convencional (PC) com $34 \mathrm{mg} \mathrm{C} \mathrm{Kg}^{-1}$ de solo seco (Figura $\mathrm{A}$ ). Os demais sistemas de cultivo não apresentaram diferença significativa com o PC apresentando valores de 47,8, 50,5 e 54,8 mg C Kg-1 de solo seco para os sistemas de cultivo agroflorestal (PAF), cultivo orgânico $(\mathrm{PO})$ e cultivo orgânico em transição para biodinâmico (POT), respectivamente (Figura ıA). Esses resultados corroboram com Kandeler et al. (1999), já que solos cultivados apresentaram menores teores de CBM do que os solos não cultivados. Em trabalhos comparativos entre sistemas de produção orgânico e convencional (MALUCHE-BARRETA et al., 2007; CAREY et al., 2009; CANALI et al., 2009), os valores de CBM foram superiores nos sistemas orgânicos em relação ao convencional devido ao maior aporte de resíduos orgânicos via adubação e manutenção de vegetação espontânea ou cultivada nas entrelinhas. O CBM é um indicador sensível ao manejo dado ao solo por representar a fração viva do solo (MOREIRA e SIQUEIRA, 
2006), mas, em pomares, pode não haver diferença mesmo entre sistemas de produção, dado a inexistência do revolvimento do solo.
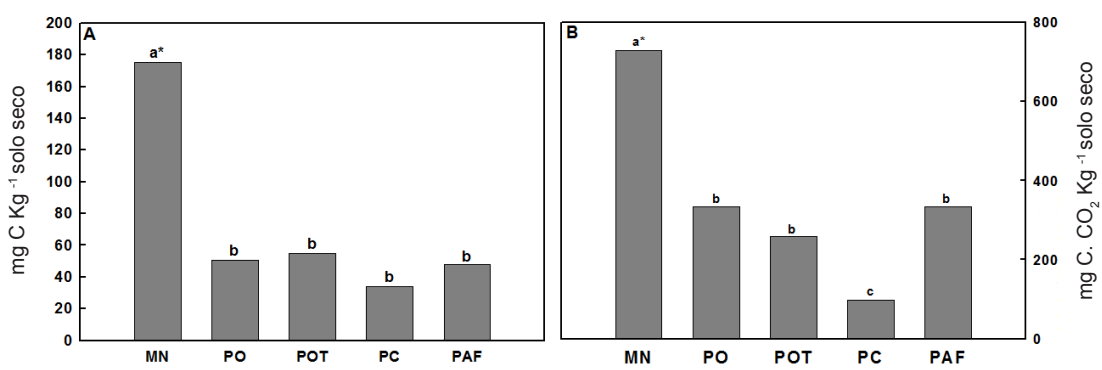

Figura 1. Carbono da biomassa microbiana (A) e respiração basal (B) dos solos sob mata nativa $(M N)$ e sob diferentes tipos de cultivo de tangerina: cultivo orgânico (PO), cultivo orgânico em transição para biodinâmico (POT), cultivo agroflorestal (PAF) e cultivo convencional (PC). "Médias seguidas de mesma letra não diferem entre si pelo teste $t$ de Student a $5 \%$ de probabilidade.

Os valores mais altos da RB foram encontrados na MN com 728,7 mg C- $\mathrm{CO}_{2} \mathrm{~kg}^{-1}$ solo seco após 20 dias de incubação (Figura 1B). Já os sistemas de cultivo PAF (334,4 mg C-CO $\mathrm{kg}^{-1}$ solo seco), PO (333,3 mg C-CO $\mathrm{kg}^{-1}$

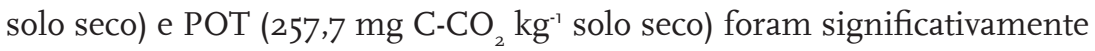
menores ao tratamento controle $(\mathrm{MN})$, embora tenham apresentado valores significativamente maiores ao sistema de cultivo PC, que obteve RB de 97,0 mg C- $\mathrm{CO}_{2} \mathrm{~kg}^{-1}$ solo seco após 20 dias de incubação (Figura $1 \mathrm{~B}$ ). Segundo Anderson (1982), a RB representa a atividade microbiana do solo pela atividade deles em oxidar a matéria orgânica do solo (MOS). Este resultado pode ser explicado pelo teor de MOS encontrado no sistema PC que foi de $2 \%$, sendo que os demais sistemas apresentaram valores próximos a $4 \%$ (Tabela 1). A maior RB do solo da MN pode ser explicada pelo maior aporte de resíduos orgânicos e pelo equilíbrio do ecossistema. Silveira (2007) verificou que a maioria dos solos cultivados apresentou menores valores de RB que os solos de vegetação nativa. Porém, outros autores não verificaram diferença na RB de solos sob manejo orgânico e convencional de pomares de maçã, no Estado de Santa Catarina (MALUCHE-BARETTA, 2007), e de kiwi, na Nova Zelândia (CAREY et al., 2009), com relação aos solos não cultivados. As ações antrópicas causam estresses, levando a população microbiana a mudanças na sua composição e em atividades metabólicas específicas (MOREIRA e SIQUEIRA, 2006), podendo alterar positivamente ou negativamente a respiração basal. 
As maiores concentrações da atividade de urease foram encontradas no tratamento controle (MN), com valores $128 \mu \mathrm{g} \mathrm{N}-\mathrm{NH}_{4} \mathrm{~g}^{-1}$ solo seco $2 \mathrm{~h}^{-1}$ (Figura 2). Os sistemas POT e PO foram significativamente inferiores, apresentando valores de 77,7 e 68,5 $\mu \mathrm{g} \mathrm{N}^{-\mathrm{NH}_{4}} \mathrm{~g}^{-1}$ solo seco $2 \mathrm{~h}^{-1}$, respectivamente. Os valores mais baixos foram encontrados nos sistemas de cultivo de tangerinas PAF e PC, com valores de 51,9 e 48, $1 \mu \mathrm{g} \mathrm{N}-\mathrm{NH}_{4} \mathrm{~g}^{-1}$ solo seco $2 \mathrm{~h}^{-1}$, respectivamente (Figura 2). De acordo com Longo e Melo (2005), em condições tropicais, pouco se conhece sobre a atividade da urease no solo e os fatores que a alteram. Pode-se constatar que o fato de a maior atividade da urease ser na área de MN está de acordo com outros autores (LONGO e MELO, 2005; PANCHOLY e RICE, 1972), os quais mostraram que áreas com maior quantidade de material orgânico e diversidade da vegetação influenciam numa maior atividade dessa enzima. Porém, no sistema PAF, a menor atividade da urease não era esperada, visto que, nesse sistema, o manejo é menos intensivo e há maior diversidade da flora na área.

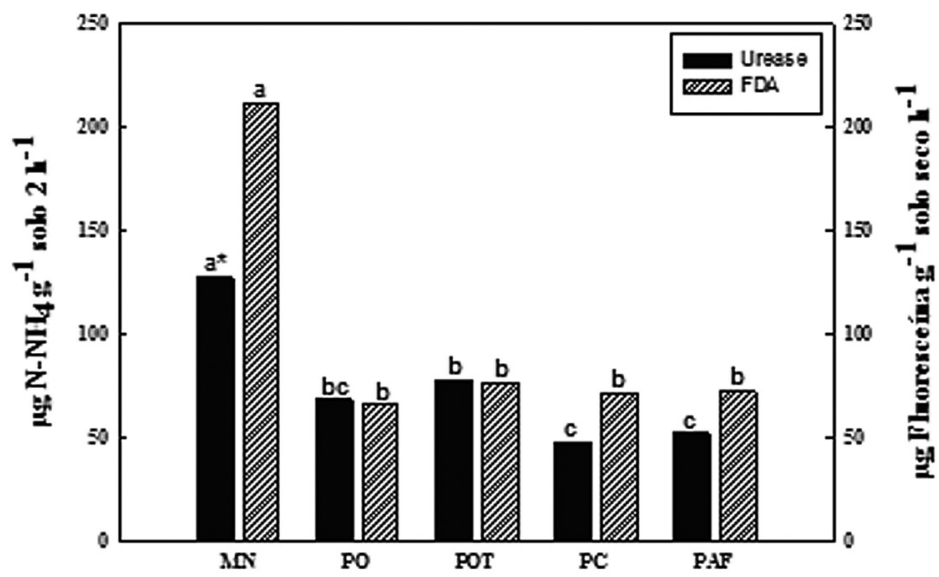

Figura 2. Atividade de urease e hidrólise do diacetato de fluoresceína (FDA) dos solos sob mata nativa $(\mathrm{MN})$ e sob diferentes tipos de cultivo de tangerina: cultivo orgânico $(\mathrm{PO})$, cultivo orgânico em transição para biodinâmico (POT), cultivo agroflorestal (PAF) e cultivo convencional (PC).

A determinação da hidrólise do diacetato de fluoresceína (FDA) é utilizada como indicador geral da atividade hidrolítica, para quantificar as células ativas nos solos e para caracterizar a atividade microbiana global do 
solo (GREEN et al., 2006). Em nosso estudo, o tratamento controle (MN) mostrou o maior valor na determinação da atividade FDA com $212 \mu \mathrm{g} \mathrm{F} \mathrm{g}^{-1}$ solo seco h- (Figura 2). Entre os sistemas de cultivo POT (76 $\mu \mathrm{g} \mathrm{F} \mathrm{g-1} \mathrm{solo}$ seco $\left.\mathrm{h}^{-1}\right)$, PAF (72 $\mu \mathrm{g} \mathrm{F} \mathrm{g}^{-1}$ solo seco $\left.\mathrm{h}^{-1}\right)$, PC (71 $\mu \mathrm{g} \mathrm{F} \mathrm{g}^{-1}$ solo seco $\left.\mathrm{h}^{-1}\right)$ e PO (66 $\mu \mathrm{g} \mathrm{F} \mathrm{g}^{-1}$ solo seco $\mathrm{h}^{-1}$ ), não houve diferença significativa, sendo que todos foram significativamente menores ao tratamento controle (MN) (Figura 2). Segundo Carvalho (2005), a alta atividade heterotrófica é esperada em solos de mata, pois esta apresenta grande deposição de material orgânico no solo, servindo de fonte de nutrientes para os microrganismos.

Para a atividade da fosfatase ácida (FA), os maiores valores encontrados foram de $1419 \mu \mathrm{g}$ p-nitrofenol $\mathrm{g}^{-1}$ solo seco $\mathrm{h}^{-1}$ no tratamento controle (MN) (Figura 3). Para os sistemas de cultivo PC e PAF, os valores da atividade de FA obtidos foram quase três vezes menores $(541$ e $427 \mu \mathrm{g}$ p-nitrofenol $\mathrm{g}^{-1}$ solo seco $\mathrm{h}^{-1}$, respectivamente) quando comparados com o tratamento controle (MN). Os menores valores de FA encontrados nos sistemas de cultivo de tangerina foram nos sistemas POT e PO, com valores de 279 e $270 \mu \mathrm{g}$ p-nitrofenol g-1 solo seco $\mathrm{h}^{-1}$, respectivamente.

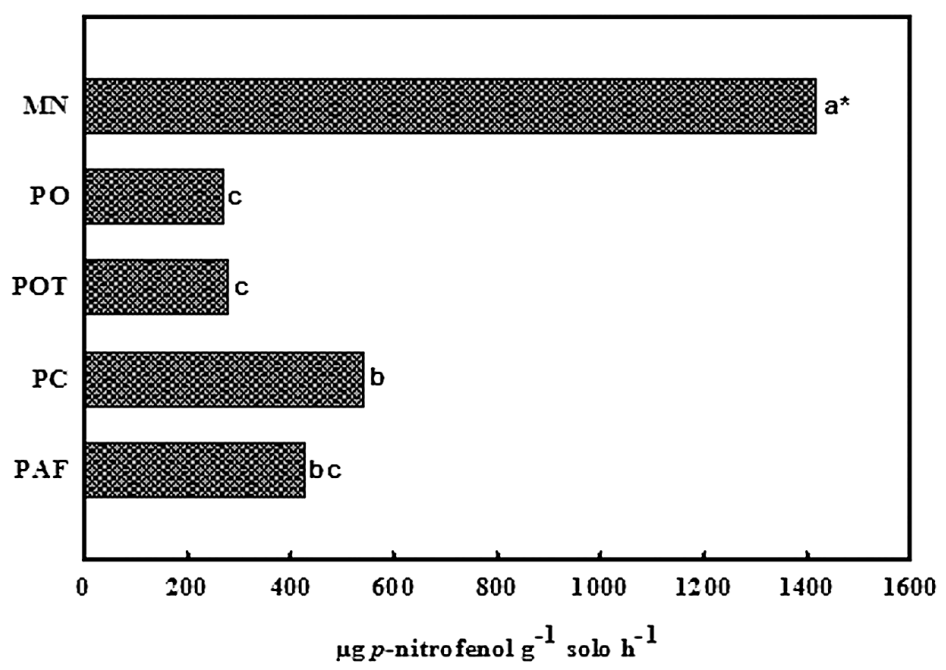

Figura 3. Atividade fosfatase ácida dos solos sob mata nativa (MN) e sob diferentes tipos de cultivo de tangerina: cultivo orgânico (PO), cultivo orgânico em transição para biodinâmico (POT), cultivo agroflorestal (PAF) e cultivo convencional (PC). "Médias seguidas de mesma letra não diferem entre si pelo teste $t$ de Student a $5 \%$ de probabilidade. 
A produção da enzima fosfatase é favorecida pela baixa disponibilidade de fósforo $(\mathrm{P})$ às plantas e aos microrganismos, podendo ser inibida por altas concentrações de fosfato inorgânico no solo (STEGE et al., 2009). Isso corrobora com os teores de fósforo apresentados nos solos (Tabela 1), cujos valores variam entre 17 e $>100$, sendo que o solo do tratamento controle (MN) apresenta o menor teor (17 $\left.\mathrm{mg} \mathrm{kg}^{-1}\right)$, seguido pelo PAF (51 $\left.\mathrm{mg} \mathrm{kg}^{-1}\right)$, PC (92 mg $\left.\mathrm{kg}^{-1}\right)$, PO (>100 $\left.\mathrm{mg} \mathrm{kg}^{-1}\right)$, e POT (>100 $\left.\mathrm{mg} \mathrm{kg}^{-1}\right)$. A alta carga de fertilizantes orgânicos anualmente empregada nos pomares sob sistemas de cultivo PO e POT, ricos em fósforo (Tabela 2), possivelmente, diminuiu a atividade da fosfatase ácida. É preciso considerar que a atividade da fosfatase ácida reflete tanto a contribuição de microrganismos como de exsudatos radiculares, sendo maior em solos com cobertura vegetal (DICK et al., 1996), porém os resultados não mostraram aumento da atividade da fosfatase ácida nos pomares que mantêm a cobertura vegetal, já que o sistema PC teve maior atividade da fosfatase ácida que os sistemas PO e POT.

Indicadores de qualidade do solo como a respiração basal, a fosfatase ácida e a urease podem ser indicadores importantes na avaliação da qualidade ambiental de diferentes sistemas de cultivo em pomares de tangerina, e devem ser mais estudados para tentar avaliar e reduzir o impacto ambiental da agricultura e das técnicas de cultivo, como diferentes sistemas de cultivo, aplicação de resíduos e adubação mineral.

\section{Conclusão}

Em todos os atributos qualitativos dos solos avaliados, a área de mata nativa mostra os maiores valores dos indicadores estudados. Isso evidencia que, apesar de qualquer cultivo da tangerina com a menor utilização de insumos, como no caso do cultivo orgânico, todos alteram a qualidade microbiológica do solo, quando comparado a um sistema natural (mata nativa) ou com a baixa interferência antrópica. Contudo, verificou-se que o cultivo convencional é o sistema que altera com maior intensidade a qualidade biológica do solo, como pôde ser verificado a partir dos resultados da biomassa microbiana, da respiração basal e da atividade da urease nos solos sob cultivo de tangerina. 


\section{Referências}

ANDERSon, J.P.E. Soil Respiration. In: PAGE, A.L., MILLER, R.H., KEENEY, D.R. (eds.). Method of analysis. 2 ed. Part 2. Madison: American Society of Agronomy: Soil Science Society of Agronomy, 1982. p.831-871.

ARAÚJO, A.S.F.; MONTEIRO, R.T.R. Indicadores biológicos de qualidade do solo.

Bioscience Journal, Uberlândia, v. 23, n. 3, p. 66-75, 2007.

BRASIL. Lei $\mathbf{n}^{\circ} \mathbf{1 0 . 8 3 1}$, de 23 de dezembro de 2003. Dispõe sobre a agricultura orgânica e dá outras providências. Diário Oficial da União, Brasília, 24 de dezembro de 2003 , Seção 1, p.8.

BUZINARO, T.N.; BARBOSA, J.C.; NAHAS, E. Atividade microbiana do solo em pomar de laranja em resposta ao cultivo de adubos verdes. Revista Brasileira de Fruticultura, Jaboticabal, v.31, n.2, p.408-415, 2009.

CANALI, s. et al. Effect of different management strategies on soil quality of citrus orchards in Southern Italy. Soil Use and Management, London, v.25, p.34-42, 2009. CAREY, P.L.; BENGE, J.R.; HAYNES, R.J. Comparison of soil quality and nutrient budgets between organic and conventional kiwifruit orchards. Agriculture, Ecosystems and Environment, Amsterdam, v.132, p.7-15, 2009.

CARVALHO, F. Atributos bioquímicos como indicadores da qualidade de solo em floresta de Araucária angustifólia (Bert). 2005. 79f. Dissertação (Mestrado em Ciência do Solo) - Escola Superior de Agricultura Luiz de Queiroz, Piracicaba. D'ANDREA, A.F.D.; SILVA, M.L.N.; CURI, N.; SIQUEIRA, J.O.; CARNEIRO, M.A.C. Atributos biológicos indicadores da qualidade do solo em sistemas de manejo na região do cerrado no sul do estado de Goiás. Revista Brasileira de Ciência do Solo, Viçosa, v.26, p.913-923, 2002.

DICK, R.P.; BREACKWELL, D.P.; TURCO, R.F. Soil enzyme activities and biodiversity measurements as integrative microbiological indicators. In: DORAN, J.W.; JONES, A.J. (eds). Methods for assessing soil quality. Madison: SSSA, 1996. p.247-271. GAMA-RODRIGUES, E.F.; GAMA-RODRIGUES, A.C.; BARROS, N.F. Biomassa de carbono e de nitrogênio de solos sob diferentes coberturas vegetais. Revista Brasileira de Ciência do Solo, Viçosa, v.21, p.361-365, 1997. GLIESSMAN, S. R. Agroecologia: processos ecológicos em agricultura sustentável. 2 ed. Porto Alegre : Ed. Universidade/UFRGS, 2001. 653p. 
GREEN, V.S.; STOTT, D.E.; MIACK, M. Assay for fluorescein diacetate hydrolytic activity: Optimization for soil samples. Soil Biology and Biochemistry, Oxford, v.38, n.3, p.693-701, 2006.

GRUPEX. O Cultivo dos Citros no Rio Grande do Sul: Referências Tecnológicas. Porto Alegre : FEPAGRO, 2005. 141p.

HAmm, A. A citricultura dentro do Programa Estadual de Fruticultura PROFRUTA. In: XII Ciclo de Palestras sobre Citricultura do RS. 2005. Anais...Faxinal do Soturno, RS: CIPACITRI, 2005. 140 p.

KANDELER, E.; TSCHERKO, D.; SPIEGEL, H. Long-term monitoring of microbial biomass, $\mathrm{N}$ mineralization and enzyme activities of a Chernozem under different tillage management. Biology and Fertility of Soils, Heidelberg, v.28,p.343-351, 1999.

LONGO, R.M.; MELO, w J. Atividade da urease em latossolos sob influência da cobertura vegetal e da época de amostragem. Revista Brasileira de Ciência do Solo, Viçosa, v.29, p.645-650, 2005.

MALUCHE-BARETTA, C.R.D. et al. Atributos microbianos e químicos do solo em sistemas de produção convencional e orgânico de maçãs no Estado de Santa Catarina. Revista Brasileira de Ciência do Solo, Viçosa, v.31, p.655-665, 2007. MOREIRA, F.M.S.; SIQUEIRA, J.O. Microbiologia e Bioquímica do Solo. 2 ed. Lavras: Editora UFLA, 2006. 729p.

PANZENHAGEN, N.v. et al. Aspectos técnico-ambientais da produção orgânica na região citrícola do Vale do Rio Caí, RS. Ciência Rural, Santa Maria, v.38, p.90-95, 2008. PANCHOLY, S.K.; RICE, E.L. Effect of storage conditions on actives of urease, invertase, amylase and dehydrogenase in soil. Soil Science Society of America, New York, v.36, p.536-537, 1972.

RIENZI, E.A.; MAGGI, A.E. Manejo y conservación de suelos de sistemas frutícolas. In: SOZZI, G. (Ed. Lit.). Árboles Frutales: Ecofisiología, Cultivo y Aprovechamiento. 1. ed. Buenos Aires: Editorial Facultad de Agronomía Universidad de Buenos Aires, 2007. p.343-362.

SANTOS, V. B. et al. Biomassa, atividade microbiana e teores de carbono e nitrogênio totais de um Planossolo sob diferentes sistemas de manejo. Revista Brasileira de Agrociência, Pelotas, v.10, p.333-338, 2004. 
SILVEIRA, A.O. Atividades enzimáticas como indicadores biológicos da qualidade de solos agrícolas do Rio Grande do Sul. 2007. 94f. Dissertação (Mestrado em Ciência do Solo) - Faculdade de Agronomia, Universidade Federal do rio Grande do Sul, Porto Alegre.

SORRENTI, G.B. et al. Influência da adubação orgânica no crescimento de tangerina cv. Clemenules e nos atributos químicos e microbiológicos do solo. Revista Brasileira de Fruticultura, Jaboticabal, v.30, n.4, p.1129-1135, 2008.

STATSOFT, INC. Statistica (data analysis software system), version 7. 2007. $<$ www.statsoft.com>

STEGE, P.W. et al. Determination of arylsulphatase and phosphatase enzyme activities in soil using screen-printed electrodes modified with multi-walled carbon nanotubes. Soil Biology and Biochemistry, Oxford, v.41, p.2444-2452, 2009 .

STOTZKY, G. Activity, ecology and population dynamics of microorganisms in soil quality. Critical Reviews in Microbiology, Boca Raton, v.1, p.59-137, 1972. STRECK, E.V. et al. Solos do Rio Grande do Sul. 2.ed. Porto Alegre: Emater/RS, 2008. 222p.

TATE, K.R.; ROSS, D.J;; FELTHAM, C.W. A direct extraction method to estimate soil microbial C: Effects of experimental variables and some different calibration procedures. Soil Biology and Biochemistry, Oxford, v.20, p.329-335, 1988. TEDESCO, M.J. et al. Análises de solo, plantas e outros materiais. 2. ed. Porto Alegre : Departamento de Solos, Faculdade de Agronomia, UFRGS. 1995. 174p. (Boletim Técnico de Solos, 5).

Submetido em: 20/12/2010

Aceito em: 29/11/2011 